\title{
Martin Puchner: The Language of Thieves. My Family's Obsession with a Secret Code the Nazis Tried to Eliminate
}

New York: W.W. Norton 2020 - ISBN 978-1324005919-26,95 \$

https://doi.org/10.14361/zig-2021-120218

1974 erschien ein Buch von großer Merkwürdigkeit. In Günter Puchners Kundenschall findet man eine Fülle von Texten, die nicht etwas aus der, sondern im Gegenteil in die vielleicht obskurste sprachliche Varietät übersetzt sind, die die deutschsprachige kulturelle Sphäre zu bieten hat: ins Rotwelsch, eine auf der deutschen Grammatik gründende Sondersprache, deren Wörter aus dem Jiddischen, dem Romanes sowie romanischen, germanischen und slawischen Sprachen stammen. "Sachsolm, Sachsolm / über hackel, / über hackel in dem Zund" liest man da etwa als Übersetzung der ersten Strophe von Hoffmanns von Fallersleben Lied der Deutschen (Puchner 1974: 129; Hervorh. i.O.). Günter Puchners Neffe Martin Puchner, der an der Harvard University englische und vergleichende Literaturwissenschaft lehrt, hat dem, was er die Obsession seiner Familie mit Rotwelsch nennt, mit The Language of Thieves ein Buch gewidmet - ein so streitbares wie brillantes Buch, das eine lange fällige Korrektur der wissenschaftlichen Perspektive auf Rotwelsch vornimmt und die kulturelle Bedeutung dieser Sprache und ihrer Verfolgung in innovativer Weise herausarbeitet. Das Buch ist im Herbst 2021 in deutscher Übersetzung unter dem Titel Die Sprache der Vagabunden. Eine Geschichte des Rotwelsch und das Geheimnis meiner Familie erschienen (Puchner 2021).

Puchners Buch ist keine wissenschaftliche Monographie im klassischen Sinne. Es bewegt sich zwischen autobiographischer Reflexion, Erforschung der eigenen Familiengeschichte und einer als detektivisch inszenierten Rotwelschforschung. Es ist diese komplexe und in einigen Zügen semiliterarische Konstellation, die Puchner zu seinem innovativen Perspektivwechsel führt. Ausgangspunkt der Reise, auf die er uns mitnimmt, ist seine Entdeckung, dass der Großvater Karl Puchner 1934 einen sprachgeschichtlichen Aufsatz veröffentlicht hatte, in dem er mit einem völkisch-antisemitischen Erkenntnisinteresse die Unterscheidung sarischer von sjüdischen N Namen zu begründen versuchte. Die in diesem $\mathrm{Zu}$ sammenhang von Karl Puchner geäußerten Hasstiraden gegen das Jiddisch und die Assoziation von Jiddisch und Rotwelsch, die Karl Puchner vornimmt, bilden eine Dissonanz zu Martin Puchners wie selbstverständlicher Begegnung mit Rotwelsch und Rotwelsch-Sprecherinnen in seiner Kindheit, die den ersten Kern des Forschungsinteresses bildet.

Nach dem Kindheitsvorspiel und der die Forschung auslösenden Entdeckung in der Widener Library der Harvard University (vgl. Kap. 1) setzen die Ausführungen im zweiten Kapitel zunächst einmal da ein, wo viele Darstellungen der Geschichte des Rotwelsch beginnen, bei der wirkmächtigsten der frühen Aufzeichnungen des Rotwelsch im anonymen Liber vagatorum (1510). Puchner stellt den Liber vagatorum konsequent in den Kontext der Frühreformation, interessiert sich also in erster Linie für Martin Luthers Neuherausgabe von 1528. Für Luther stehe Rotwelsch, so argumentiert Puchner, im Zusammenhang der Kultivierung einer deutschen Schrift- 
sprache, die für Kirchenlied und Bibel geeignet war. Eine hybride Sprachform, wie sie der Liber vagatorum zum Zwecke der Warnung präsentierte, musste Luther, so argumentiert Puchner, nicht nur verdächtig sein, sie erschien geradezu als Widersacherin der eigenen Bemühungen um das Deutsche. In seiner Vorrede zur Neuausgabe des Liber vagatorum folgert Luther aus der Häufung von aus dem Hebräischen stammenden Wörtern, die "rottwelsche sprache« sei »von den Juden komen « (Luther 1909: 638), ${ }^{1}$ was im Horizont des Luther'schen Antijudaismus eine vollumfängliche Verurteilung bedeutet. Doch Puchner macht an dieser Stelle eindrücklich auf den Umstand aufmerksam, dass die einhellige Verdammung des Rotwelsch in den gedruckten Quellen der Frühen Neuzeit keineswegs den Versuch zur Konsequenz hatte, es zum Verschwinden zu bringen. Im Gegenteil: Die Quellen seit dem Liber vagatorum zeichnen nicht nur rotwelsches Wortmaterial auf, um, so das Argument, Betrügereien vorzubeugen, sie beginnen paradoxerweise, im Falle des Liber vagatorum im Rahmen der Beschreibung von Bettlertypen, selbst Rotwelsch zu sprechen. So heißt es dort etwa in der Beschreibung des Bettlertyps der »Stabü le[r]«: »vnd hond den wetterhan vnd den wintfang vol zeichen hangen von allen heiligen, vnnd ist der wintfang gevetzt von allen stucken, vnd hant dann die hutzen die yn den lehem dippen « (zit. n. Kluge 1987: 38). ${ }^{2}$ In dem historischen Ausblick, der das Kapitel beschließt, deu-

1 »[R]otwelsche Sprache«, »von den Juden gekommen« (Übers. B.W.).

2 "Und haben den Wetterhahn und den Windfang, den Hut und den Mantel voll Zeichen hängen, und der Windfang ist gefetzt, der Mantel ist gearbeitet aus vielen Stücken. Und die haben dann die Hutzen, die ihnen den Lehem dippen, die Bauern, die ihnen Brot geben« (Liber vagatorum 1987: 81; Hervorh. i.O.). tet sich Puchners These über Rotwelsch an: Mit dem Ende des Dreißigjährigen Krieges habe sich Rotwelsch von einer professionellen Zweitsprache bestimmter sozialer Gruppen zu einer Sprache entwickelt, die "something closer to the native tongue of those growing up amid these large roaming gangs « (35) sei. Auf welche Quellen oder historiographische Darstellungen er sich für diese These bezieht, legt Puchner leider nicht offen.

Nach dem Blick auf die Anfänge der Auseinandersetzung mit Rotwelsch in gedruckten Quellen wendet sich das dritte Kapitel erneut der Puchner'schen Familiengeschichte $\mathrm{zu}$. Dieses Ineinander von Rotwelsch- und Familiengeschichte, diese Ausgestaltung der Zweizeitigkeit der Forschung, macht eine zentrale Faszination des Buches aus. Konfrontiert mit der Entdeckung Martin Puchners über seinen Großvater erzählt der Vater, wie er und sein Bruder Günter auf einer Fotografie das Parteiabzeichen am Revers Karl Puchners entdeckten. Diese Entdeckung kontextualisiert Martin Puchner in der deutschen sogenannten Vergangenheitsbewältigung. Damit stellt er die Rotwelschforschung mehr, als das jemals vor ihm geschehen ist, mit kritischer Stoßrichtung in den Kontext der Geschichte der deutschen Nation und des für sie konstitutiven Antisemitismus. Der Umstand, dass sich in der Auseinandersetzung von Nicht-Rotwelsch-Sprecherinnen mit dem Rotwelsch durchgehend auch ein für die deutsche Nation ebenfalls konstitutiver Antiziganismus (vgl. Patrut 2014) artikuliert, schlägt sich dagegen in Puchners Buch eher in Randbemerkungen nieder. Das ist vor dem Hintergrund des Umstands erklärungsbedürftig, dass erst mit Johann Christoph Christian Rüdigers Nachweis der Verwandtschaft des Romanes mit dem Sanskrit 1782 zwischen >Gaunersprache und Zigeunersprache $\mathrm{zu}$ unterscheiden 
begonnen wurde (vgl. Bogdal 2011: 154159). Noch 1897 lässt Jakob Wassermann seine "Zigeuner in ihrem Rotwälsch" sprechen (Wassermann 1918: 37).

Das folgende Kapitel wendet sich wieder - aus Anlass der Rotwelschforschungen des Onkels Günter Puchner - der Geschichte des Rotwelschdiskurses zu. Fokussiert werden hier die polizeilichen Bemühungen um die Aufzeichnung des Rotwelsch, wie sie das 19. Jahrhundert kennzeichnen. Dies schließt eine kritische Würdigung vor allem der Bemühungen Friedrich Christian Benedict Avé-Lallemants und seines Werks über Das deutsche Gaunerthum (1858-1862) ein. Puchner macht die Ambivalenz dieser ersten umfangreichen Rotwelschforschung mit wissenschaftlichem Anspruch deutlich, wenn er sowohl die Wendung gegen das Rotwelsch, das Avé-Lallemant als "hostile force" (68) angesehen habe, als auch die Sorgfalt seines Unterfangens herausstellt, Rotwelsch als historisches Phänomen ernst zu nehmen.

Im selben Kapitel wendet sich Puchner schließlich einem überraschenden Text zu: Adolf Hitlers Mein Kampf, das er im Archiv des Onkels findet. An dieser für die Zuspitzung des deutschen Antisemitismus bedeutsamen Stelle findet Puchner die Rede vom Jiddisch als einer Geheimsprache auf, die, wie er erläutert, den nichtjüdischen Diskurs über das Jiddisch seit langem begleitet hatte. Von hier aus stellt sich die Frage: Ist Rotwelsch eine Geheimsprache, wie es der Diskurs von Nicht-Rotwelsch-Sprechern seit mindestens dem Liber vagatorum und wie es die sprachwissenschaftliche (vgl. Siewert 2003: 15f.) und populärwissenschaftliche (vgl. Girtler 2019: 26) Rotwelschforschung bis heute behauptet? Oder ist der Vorwurf >Geheimsprache vielmehr eine antisemitische (und, so wäre über Puchner hinaus $\mathrm{zu}$ fragen, antiziganistische) Belastung, die es zu kritisieren gilt, um einen angemesseneren Blick auf das Rotwelsch zu ermöglichen?

Diese Frage bestimmt zunehmend die weiteren Kapitel des Buches, deren Themen ich hier nur knapp nennen kann: die Selbstorganisation von >Vagabunden um Gregor Gog in der Weimarer Republik und die für die Polizei angefertigten Aufzeichnungen Carl Heinrich Ferdinand Baumhauers von der Mitte des 19. Jahrhunderts - Aktivitäten und Äußerungen von Rotwelsch-Sprechern selbst (Kap. 5); eine mündliche Familienüberlieferung aus der Familie von Martin Puchners Mutter über den Großvater und dessen KZ-Aufenthalt - und deren realer Hintergrund (Kap. 6); eine Reise des Vaters nach Prag als Anlass, über die Rotwelschbezüge Franz Kafkas und Gustav Meyrinks nachzudenken (Kap. 7); Günter Puchners Kundenschall und sein Lyrikband Ein Arm voll Schmonzes (1983; Kap. 8); die Bemühungen des Esperanto-Erfinders Ludwig Zamenhof um die Kodifizierung der Sprache (Kap. 9); Karl Puchners Karriere im Bayerischen Staatsarchiv vor und nach 1945 (Kap. 10); dessen Bruder Ottos Karriere im Staatsarchiv Nürnberg (Kap. 11); die Erzählung von der Einbürgerung Martin Puchners in die USA als Anlass, über Unterschiede im Selbstverständnis der US-amerikanischen und der deutschen Nation nachzudenken (Kap. 12); ein Besuch der Tante Roswitha Puchner in New York (Kap. 13); Spuren von Rotwelsch in den USA (Kap. 14). Abgeschlossen wird das Buch von einem Kapitel, das von der Suche nach Kontakt mit Schweizer Jenischen erzählt.

Puchners Buch führt drei wesentliche Innovationen in die Rotwelschforschung ein: Die Forschung bringt ihn erstens dazu, die Charakterisierung von Rotwelsch als Geheimsprache zu hinterfragen. Dem stellt er das Bild einer Umgangssprache bestimmter sozialer Gruppen gegenüber - besonders vulnerabler 
Gruppen, die, wie Sprecherinnen jeder Sprache es ebenfalls tun, im Gefahrenfall die Unverständlichkeit ihrer Sprache für ihre Verfolgerinnen auszunutzen. Aus dieser Sicht erscheint »the entire idea that Rotwelsch was a secret language als »something like a projection« (86). Um den Charakter des Rotwelsch begrifflich zu fassen, greift Puchner schließlich auf ein Wittgenstein'sches Sprachverständnis zurück und begreift Rotwelsch als ein "tool for survival« (230) bestimmter Bevölkerungsgruppen. Die Projektion der Rotwelschforschung, die Puchner kritisiert, steht - das ist die zweite wesentliche Einsicht - im Zusammenhang einer Geschichte der Wendung der deutschsprachigen Nicht-Rotwelsch-Sprecher gegen Rotwelsch und seine Sprecherinnen und damit auch im Zusammenhang mit der antisemitischen (und - wieder über Puchner hinaus - antiziganistischen) Ablehnung dessen, was als negatives Gegenbild des >Deutschen < imaginiert wird. Drittens aber macht Puchners Buch eindringlich deutlich, dass das abgelehnte Rotwelsch das Deutsche geradezu heimsucht (vgl. 143), was sowohl in den paranoiden Verfolgungsimpuls der nationalsozialistischen Namensforschung als auch in die Rotwelschobsession der Nachgeborenen um 1968 führt.

Von dieser Obsession ist nun das Buch selbst nicht ganz frei - und dass Puchner das auch bejahen würde, merkt man jeder Zeile des schwungvoll geschriebenen Textes an. Leider verliert das Buch im letzten Kapitel aber die kritische Haltung, die es bis dahin kennzeichnete. Dort nämlich macht sich Puchner auf die Suche nach authentischen Rotwelsch-Sprechern der Gegenwart des 21. Jahrhunderts - übrigens geleitet auch von dem 2019 in dritter Auflage erschienenen Buch von Roland Girtler (vgl. 237f.), das mit seinen Quellen unkritisch umgeht und streckenweise von naivem romantischem Antiziganismus geprägt ist (vgl. Girtler 2019). Wie sonst auch an einigen Stellen inszeniert Puchner hier eine komplizierte Forschungsbewegung. Dass, wie und wo Rotwelschdialekte aktuell gesprochen werden bzw. in den letzten 50 Jahren gesprochen wurden, wurde von der Sprachwissenschaft ausführlich erforscht - in Werken, die allesamt von Puchner nicht zitiert werden (vgl. u.a. Siewert 1996; 2003; Weiland 2003; Efing 2004; Theilacker 2017); und es braucht auch keinen Mittelsmann, um ein Treffen mit einem »Häuptling« (»Chief«, 241) anzuberaumen - »Miche would be told of the time and place of the meeting only an hour beforehand« (ebd.) -, wenn man mit Jenischen in Kontakt treten möchte. Es dürfte genügen, eine der Selbstorganisationen anzuschreiben, etwa die Schweizer Radgenossenschaft der Landstraße, ${ }^{3}$ den Jenischen Bund in Deutschland und Europa ${ }^{4}$ oder das Jenische Kulturzentrum in Singen, ${ }^{5}$ um nur einige wenige $\mathrm{zu}$ nennen. Im Verlauf des Kapitels fällt Puchner hinter den kritischen Stand, den das Buch erreicht hatte, zurück. Rotwelsch, dessen notorisches Verständnis als >Geheimspracheく er erfolgreich kritisiert hatte, erscheint nun plötzlich in einem anderen Licht: "All these years I had been looking for a term to characterize Rotwelsch, and here it was: recycling. This was the right word for a lifestyle that relied on reusing materials produced by the sedentary world [...]. Rotwelsch and its younger variants consisted exclusively of recycled words« (243). Zwar gesteht Puchner ein, dass dies eigentlich für alle Sprachen gelte, doch es bleibt dabei: »Rotwelsch and Yenish were

3 Vgl. https://www.radgenossenschaft.ch [Stand: 1.10.2021].

4 Vgl.http://www.jenische.info [Stand: 1.10.2021].

5 Vgl. https://jenisches-kulturzentrum.org [Stand: 1.10.2021]. 
recycled most fully and most self-consciously, languages that wore recycling proudly on their sleeves « (ebd.). Am Ende wird die kritische Perspektive, die das Buch so fulminant eröffnet hatte, wieder geschlossen - ein Effekt, so vermute ich, der mangelnden Reflexion auf die antiziganistischen Gehalte der Forschungsgeschichte: Rotwelsch wird hier beinahe so beschrieben, wie es immer beschrieben wurde, nämlich als Schmarotzersprache, die von Schmarotzern gesprochen wird - der Unterschied ist die positive Wendung ins nachhaltige Recycling. Die Sinnstruktur des Ressentiments aber bleibt unangestastet (vgl. End 2011), und das "Laughter of a Yenish Chief « (so die Kapitelüberschrift) muss "the unbridgeable gulf between nomadic life and the attempts on the part of sedentary, distinction-making city dwellers such as myself to understand it« (244), markieren. Am Ende sucht Puchner das (sprachlich) Nichtidentische wieder im Anderen statt das komplexe (sprachliche) Verhältnis der Nichtidentität zu fokussieren, in dem wir uns als sprachliche Wesen immer und notwendigerweise bewegen und das sich im Verhältnis zum Rotwelsch auf einzigartige Weise erforschen lässt.

Wenn es auch in diesem Punkt zu kritisieren ist, setzt Puchners Buch im Ganzen doch einen neuen Standard für eine kulturwissenschaftliche Rotwelschforschung.

Benedikt Wolf

(https://orcid.org/0000-0001-9217-3248)

\section{Literatur}

Bogdal, Klaus-Michael (2011): Europa erfindet die Zigeuner. Eine Geschichte von Faszination und Verachtung. Berlin.

Efing, Christian (2004): Jenisch unter Schaustellern. Mit einem Glossar aus schriftlichen Quellen. Wiesbaden.

End, Markus (2011): Bilder und Sinnstruktur des Antiziganismus. In: Aus Politik und Zeitgeschichte 61, H. 22-23, S. 15-21.

Girtler, Roland ( 2019$)$ : Rotwelsch. Die alte Sprache der Gauner, Dirnen und Vagabunden. Wien/Köln/Weimar.

Kluge, Friedrich (1987): Rotwelsch. Quellen und Wortschatz der Gaunersprache und der verwandten Geheimsprachen. I. Rotwelsches Quellenbuch. Nachdr. d. Ausg. Straßburg 1901. Berlin.

Liber vagatorum (1987). Der Bettler Orden. Übers. v. Heiner Boehncke und Rolf Johannsmeier. In: Dies.: Das Buch der Vaganten. Spieler, Huren, Leutbetrüger. Köln, S. 79-101.

Luther, Martin (1909): Von der falschen Bettler Büberei. In: Ders.: Werke. Kritische Gesamtausgabe. Bd. 26. Weimar, S. 634-654.

Patrut, Iulia-Karin (2014): Phantasma Nation. »Zigeuner« und Juden als Grenzfiguren des »Deutschen « (1770-1920). Würzburg.

Puchner, Günter (1974): Kundenschall[,] das Gekasper der Kirschenpflücker im Winter. Übersetzungen ins Rotwelsch v. Günter Puchner. München.

Puchner, Martin (2021): Die Sprache der Vagabunden. Eine Geschichte des Rotwelsch und das Geheimnis meiner Familie. Übers. von Matthias Fienbork. München.

Siewert, Klaus (Hg.; 1996): Rotwelsch-Dialekte. Symposion, Münster. 10. bis 12. März 1995. Wiesbaden. 
Ders. (2003): Grundlagen und Methoden der Sondersprachenforschung. Mit einem Wörterbuch der Masematte aus Sprecherbefragungen und den schriftlichen Quellen. Wiesbaden.

Theilacker, Manfred E. (2017): Der Kochemer Loschen. Die Sprache der Klugen. Zur Sozialgeschichte einer Sondersprache des Wanderhandels, der Hausierer, Bettler und Viehhändler in Württemberg. Aufgezeigt am Beispiel einer Fallstudie im Schwäbisch-Fränkischen Wald (Spiegelberg). Ostfildern.

Wassermann, Jakob (1918): Die Juden von Zirndorf [1897]. Berlin/Wien.

Weiland, Thorsten (2003): Das Hundeshagener Kochum. Ein Rotwelsch-Dialekt von Wandermusikanten aus dem Eichsfeld. Quellen - Wörterbuch - Analyse. Paderborn u.a. 
